\title{
The Effects of Tai Chi Intervention on Healthy Elderly by Means of Neuroimaging and EEG: A Systematic Review
}

\author{
Zhujun Pan ${ }^{1 *}$, Xiwen Su${ }^{2}$, Qun Fang ${ }^{1}$, Lijuan Hou ${ }^{2}$, Younghan Lee ${ }^{1}$, Chih C. Chen ${ }^{1}$, \\ John Lamberth ${ }^{1}$ and Mi-Lyang Kim ${ }^{3}$
}

${ }^{1}$ Department of Kinesiology, Mississippi State University, Starkville, MS, United States, ${ }^{2}$ Exercise Physiology Laboratory, College of Physical Education and Sports, Beijing Normal University, Beijing, China, ${ }^{3}$ Department of Sports, Leisure and Recreation, Soonchunhyang University, Asan, South Korea

OPEN ACCESS

Edited by:

Sarah A. Schoen,

STAR Institute for Sensory Processing

Disorder, United States

Reviewed by:

Xu Lei,

Southwest University, China

Deep R. Sharma,

SUNY Downstate Medical Center,

United States

*Correspondence:

Zhujun Pan

zp147@msstate.edu

Received: 09 January 2018 Accepted: 03 April 2018

Published: 18 April 2018

Citation:

Pan Z, Su X, Fang Q, Hou L, Lee Y, Chen CC, Lamberth J and Kim M-L

(2018) The Effects of Tai Chi

Intervention on Healthy Elderly by Means of Neuroimaging and EEG: $A$

Systematic Review.

Front. Aging Neurosci. 10:110.

doi: 10.3389/fnagi.2018.00110
Aging is a process associated with a decline in cognitive and motor functions, which can be attributed to neurological changes in the brain. Tai Chi, a multimodal mind-body exercise, can be practiced by people across all ages. Previous research identified effects of Tai Chi practice on delaying cognitive and motor degeneration. Benefits in behavioral performance included improved fine and gross motor skills, postural control, muscle strength, and so forth. Neural plasticity remained in the aging brain implies that Tai Chi-associated benefits may not be limited to the behavioral level. Instead, neurological changes in the human brain play a significant role in corresponding to the behavioral improvement. However, previous studies mainly focused on the effects of behavioral performance, leaving neurological changes largely unknown. This systematic review summarized extant studies that used brain imaging techniques and EEG to examine the effects of Tai Chi on older adults. Eleven articles were eligible for the final review. Three neuroimaging techniques including fMRI $(N=6)$, EEG $(N=4)$, and $M R I(N=1)$, were employed for different study interests. Significant changes were reported on subjects cortical thickness, functional connectivity and homogeneity of the brain, and executive network neural function after Tai Chi intervention. The findings suggested that Tai Chi intervention give rise to beneficial neurological changes in the human brain. Future research should develop valid and convincing study design by applying neuroimaging techniques to detect effects of Tai Chi intervention on the central nervous system of older adults. By integrating neuroimaging techniques into randomized controlled trials involved with Tai Chi intervention, researchers can extend the current research focus from behavioral domain to neurological level.

Keywords: Tai Chi, aging, neuroimaging, EEG, neural plasticity

\section{INTRODUCTION}

Older adults experience gradual regression of abilities. In addition to the physiological changes such as loss of muscular strength and declined vision, neurological ability declines with advanced aging. Tomasi and Volkow (2012) proposed that age-related decrease in motor and cognitive functions is associated with degeneration of the brain networks and changes in brain 
anatomy. Other studies indicated that decrease in functional connectivity as well as atrophy in gray matter and basal ganglia result in lack of motor control in older adults (Seidler et al., 2010; Hoffstaedter et al., 2015). However, aging process is reversible due to the plasticity and adaptivity of the human brain to experience-specific tasks (Adkins et al., 2006; Petzinger et al., 2010). Brain plasticity implies that reorganization of brain structure and functional connectivity is possible in older adults (Erickson et al., 2007). The finding suggested that appropriate intervention protocols such as exercise and motor training can counteract declines associated with advanced aging (Erickson et al., 2007; Seidler et al., 2010). For example, older adults participating in a 6-month aerobic exercise demonstrated better cardiovascular fitness and enhanced brain plasticity than the sedentary counterparts. Specifically, increased brain volume in gray and white matter were considered evidence of intact central nervous system and contributed to cognitive improvement (Colcombe et al., 2004, 2006). Bearing with the perception as to the significant role of brain plasticity in mitigating or even reversing the course of aging, researchers attempt to understand the neural mechanisms underlying exercise-related improvement in cognitive and motor performance.

Regular exercise is a practical approach to enhancing brain plasticity (Erickson et al., 2013; Voss et al., 2013). Tai Chi, a multimodal mind-body exercise integrating gracefulness, mindfulness, and gentleness, is a recommended form of physical activity for older adults (Wong et al., 2001). Benefits of practicing Tai Chi were reported in cognitive performance (Lam et al., 2011; Wayne et al., 2014) and motor functions such as postural control (Ni et al., 2014), fall prevention (Tousignant et al., 2013; Jain et al., 2017), muscle strength (Reid et al., 2016), and agility (Wayne et al., 2014). Given that neural plasticity shapes performance modification (Paré and Munoz, 2000), it is reasonable to assume that evolution of behavior associated with Tai Chi practice should be detected in the corresponding brain regions. Noninvasive neuroimaging techniques allow researchers to identify neural correlates of exercise-induced changes in the aging brain. Electroencephalography (EEG) produces spontaneous neuroelectric feedback on brain activity (Hatta et al., 2005; Fong et al., 2014). Magnetic Resonance Imaging (MRI) provides in vivo measures of brain anatomy and physiology (Giedd et al., 2015). Researchers used the technique to investigate structural changes in brain volume (Colcombe et al., 2006) and cortical thickness (Wei et al., 2013). Functional Magnetic Resonance Imaging (fMRI) detects brain connectivity based on blood oxygenation level-dependent (BOLD) signal in distinct brain regions (Fox et al., 2007). This technique has been applied to probe exercise-induced changes in brain activation and functional connection (Erickson et al., 2007; Seidler et al., 2010).

The current review summarized extant studies that applied Tai Chi to promote health for the following reasons. First, Tai Chi is an increasingly popular physical activity, which has been recommended for older adults and people with chronic disease. Second, despite the encouraging outcomes observed at the behavioral level, neural mechanisms underlying the promoted functions remain largely unknown (Voss et al., 2013). Neuroimaging (fMRI and MRI) and neuroelectric techniques
(EEG) are the instruments that expand current knowledge on the correlates between neural plasticity and modified function. In this context, we aim to investigate three main issues: (1) Tai Chi-incurred benefits in older adults; (2) improved functions and corresponding changes in the brain; and (3) the direction of future study. To our knowledge, it is the first review to systematically investigate the benefits of Tai Chi exercise from the perspective of neural plasticity. With an increasing application of neuroimaging techniques, researchers should elevate the current study of interest from mere performance to neurological level.

\section{METHODS}

\section{Literature Search}

Five electronic databases (Google scholar, PubMed, Cochrane Library, Scopus, and Web of science) were searched for relevant studies published since 1990. The following terms were entered in multiple combinations, including older adults, elderly, seniors, aging, Tai Chi Chuan, Tai chi, Taichi, and Tai Ji. Terms for neuroimaging techniques include brain imaging, electroencephalography (EEG), event-related potentials (ERP) diffuse optical tomography (DOT), diffuse optical imaging (DOI), event-related optical signal (EROS), magnetic resonance imaging (MRI), Functional magnetic resonance imaging (fMRI), diffusion tensor imaging (DTI) arterial spin labeling (ASL), magnetoencephalography (MEG), computed tomography (CT), positron emission tomography (PET), and single-photon emission computerized tomography (SPECT). Manual search was conducted for known articles in the area by titles instead of keywords search.

\section{Eligibility criteria}

Studies were eligible for inclusion if the following criteria were met: (1) subjects were healthy older adults or middle-aged adults (average age of Tai Chi group must be over 50); (2) Tai Chi was applied to exercise intervention; (3) brain imaging methods including MRI, fMRI, EEG, ERP etc. were used to assess variables of interest. The screening process consisted of two phases. First, two reviewers (XS \& ZP) independently examined title, keywords, and abstracts of retrieved articles. In the second phase, a third author (QF) was responsible to deal with any disagreement between the reviewers.

Studies that failed to conform to one of the specified criteria were considered ineligible. To gain a comprehensive understanding of Tai Chi-related changes in the central nervous system of older adults, there were no restrictions on the types of studies. However, conference abstracts, review articles, monograph, and videos were excluded.

\section{Quality Assessment}

The methodological quality was assessed by Delphi list for quality assessment (Verhagen et al., 1998). To reduce the risk of bias in assessment, two reviewers (XS \& ZP) independently scored the quality of the included articles. Inconsistencies between the two reviewers were solved after discussing with a third author. 


\section{Data Extraction}

Study characteristics encompass basic information of the selected articles, including author(s) of study and year of publication, study design, place of study, sample size and attribution rate, intervention frequency and duration, age of subjects, and measures. Age of subjects refers to the average group age, which should be above 50. Measures applied to the studies must include neuroimaging (fMRI or MRI) or neuroelectric techniques (EEG). Rationale, findings, and practical implications were summarized according to the purpose, results, and conclusions of the retrieved studies.

\section{RESULTS}

\section{Study Selection}

A total of 40 articles were retrieved from the initial search. Examination of titles and abstracts excluded 13 irrelevant articles. Further analysis of the remaining 27 items screened off 16 articles for the following reasons: lack of Tai Chi intervention $(N=10)$, participants with health issues $(N=2)$, non-journal articles $(N=2)$, lack of brain imaging method $(N=1)$, and review paper $(N=1)$. Finally, 11 studies were eligible for full-text critical appraisal. Figure 1 indicates the study selection process.

\section{Study Characteristics}

Effects of Tai Chi intervention on participants' neurological changes received an increasing attention in recent years as nine of the included studies $(N=11)$ were published in the past 5 years. China is the major country where relevant studies were conducted $(N=8)$ due to the prevalence of Tai Chi in the region. Subjects were mostly seniors. The average age of

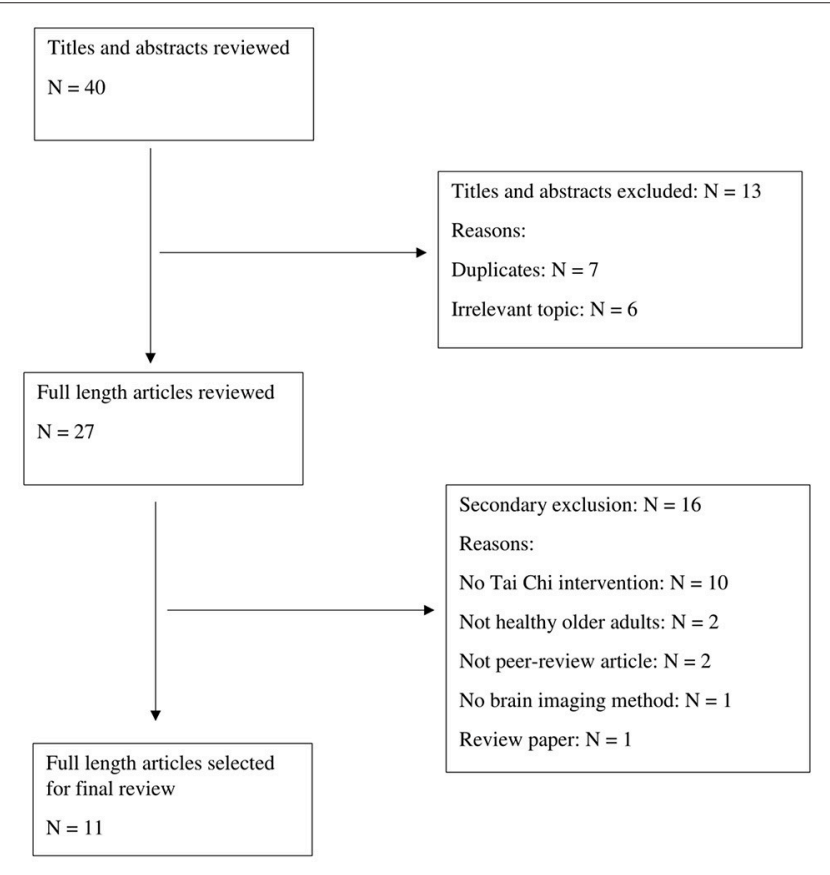

FIGURE 1 | Article selection process.
Tai Chi group in the studies ranged between 50.5 and 68.6 years. The study design included pre- and post-tests $(N=1)$, RCT $(N=5)$, and Quasi-experiment $(N=5)$. Seven studies compared the subjects' performance of Tai Chi group with that of control group before and after the intervention. The other four studies examined the difference between experienced Tai Chi practitioners and people with a relatively sedentary lifestyle.

Scales and instruments such as Attention Network Test (ANT) and Memory Scale (MS) were used to assess behavioral and cognitive performance. On the other hand, MRI, fMRI, and EEG provided evidence of neural plasticity. MRI presented the image of brain structures (Wei et al., 2013, 2014; Zheng et al., 2015). fMRI examined functional connectivity (Li et al., 2014; Tao et al., 2016, 2017) and brain neural activity (Yin et al., 2014). EEG detected the spontaneous electric activity when a subject is performing a specific task (Liu et al., 2003; Field et al., 2010; Fong et al., 2014; Hawkes et al., 2014). Combining performance assessment with neuroimaging evidence allows researchers to investigate Tai Chi-induced outcomes at both behavioral and neural levels. Study characteristics are listed in Table 1.

\section{Quality Assessment of Eligible Studies}

Most of the included studies exhibited moderate $(N=5)$ to high $(N=5)$ quality of study design, with only one being categorized as low quality. Five cross-sectional studies aimed to identify different features between experienced Tai Chi practitioners and sedentary counterparts. Participants were recruited and allocated based on Tai Chi-related experiences and thus failed to meet the requirement of random allocation. For the studies without adopting intervention protocols, criterions such as similar at baseline (SB) and therapist blinded (TB) were not applicable to the studies $(N=5)$. Details of quality assessment are listed in Table 2.

\section{Summary of Evidence}

Summary of the studies involved with four categories of interest regarding the impacts of Tai Chi on brain structures, functional connectivity, neural activity, and electric activity. Details of the summarized evidence are displayed in Table $\mathbf{1}$.

\section{Brain Structures}

One study examined the differences in the brain structures between experienced Tai Chi practitioners and people lacking routine exercise. MRI image identified thicker cortex in the left and right hemisphere of long-term Tai Chi practitioners in comparison to the cortical regions of people with a sedentary lifestyle. The study suggested that cortex thickness in the left medial occipitotemporal sulcus and lingual sulcus is subject to the intensity of Tai Chi practice (Wei et al., 2013).

\section{Functional Connectivity}

Tai Chi-induced benefits in cognitive function were observed after elderly participants receiving a 6-week multimodal intervention, which consisted of Tai Chi exercise, group counseling, and cognitive training. Changes in functional 


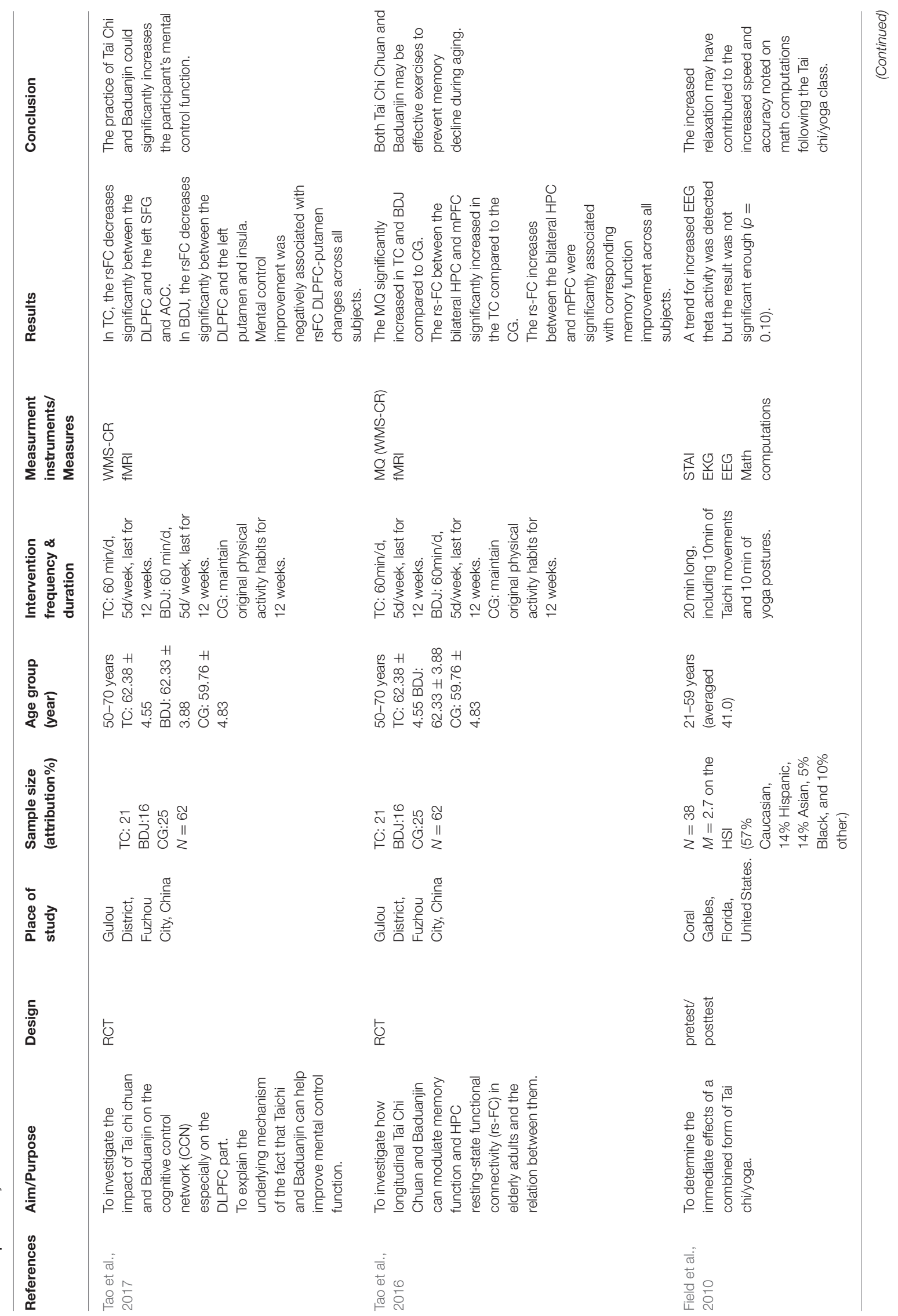



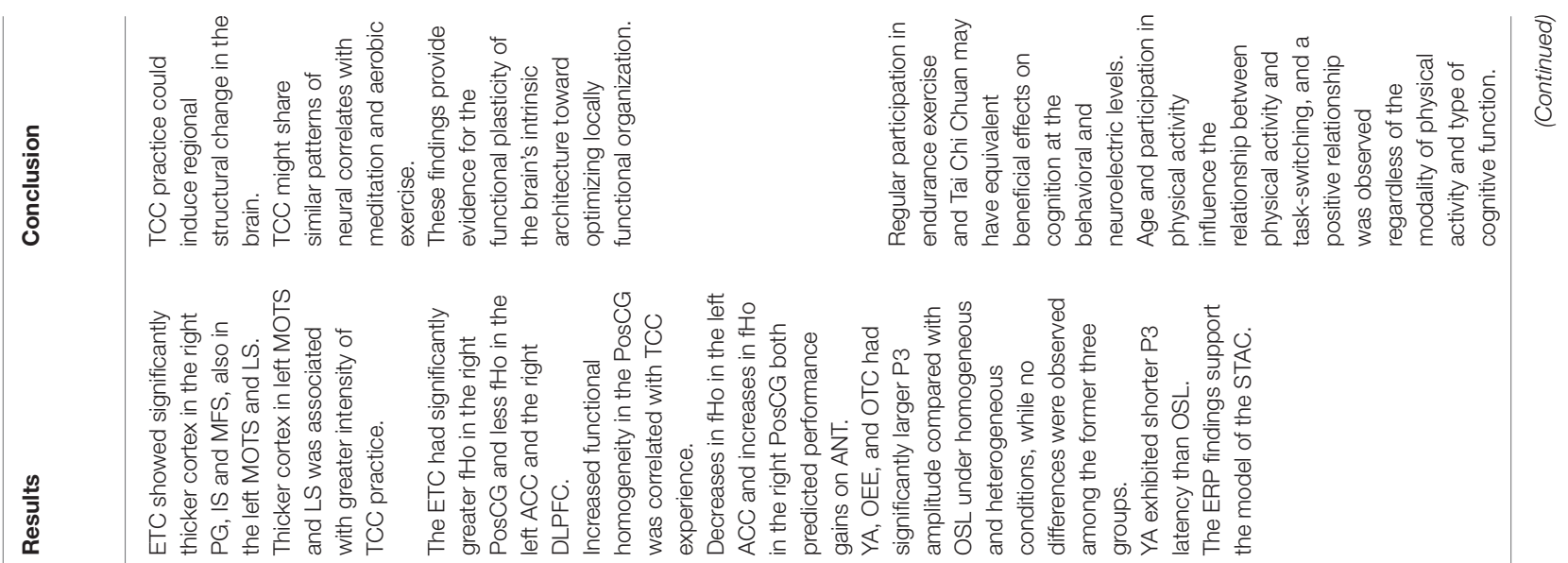

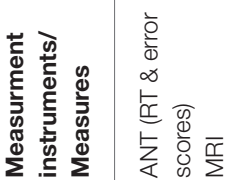

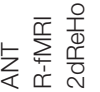

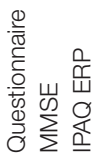

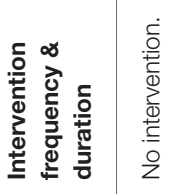

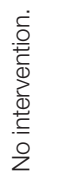<smiles>C1CCCC1</smiles>

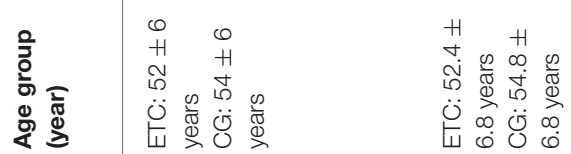

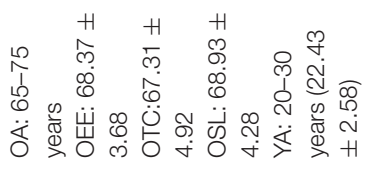

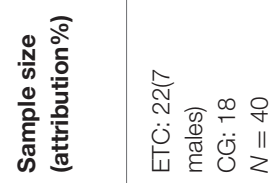

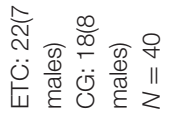

0,1
0
0

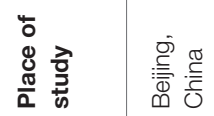

逽

赵

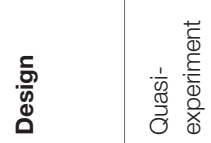

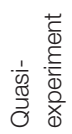

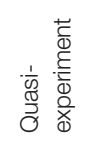
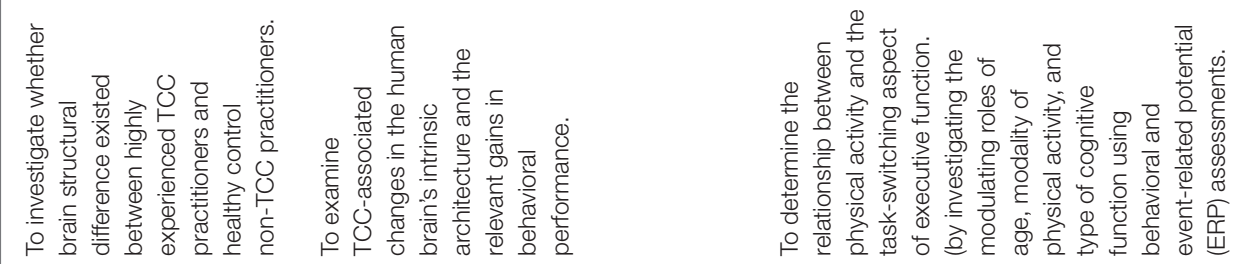


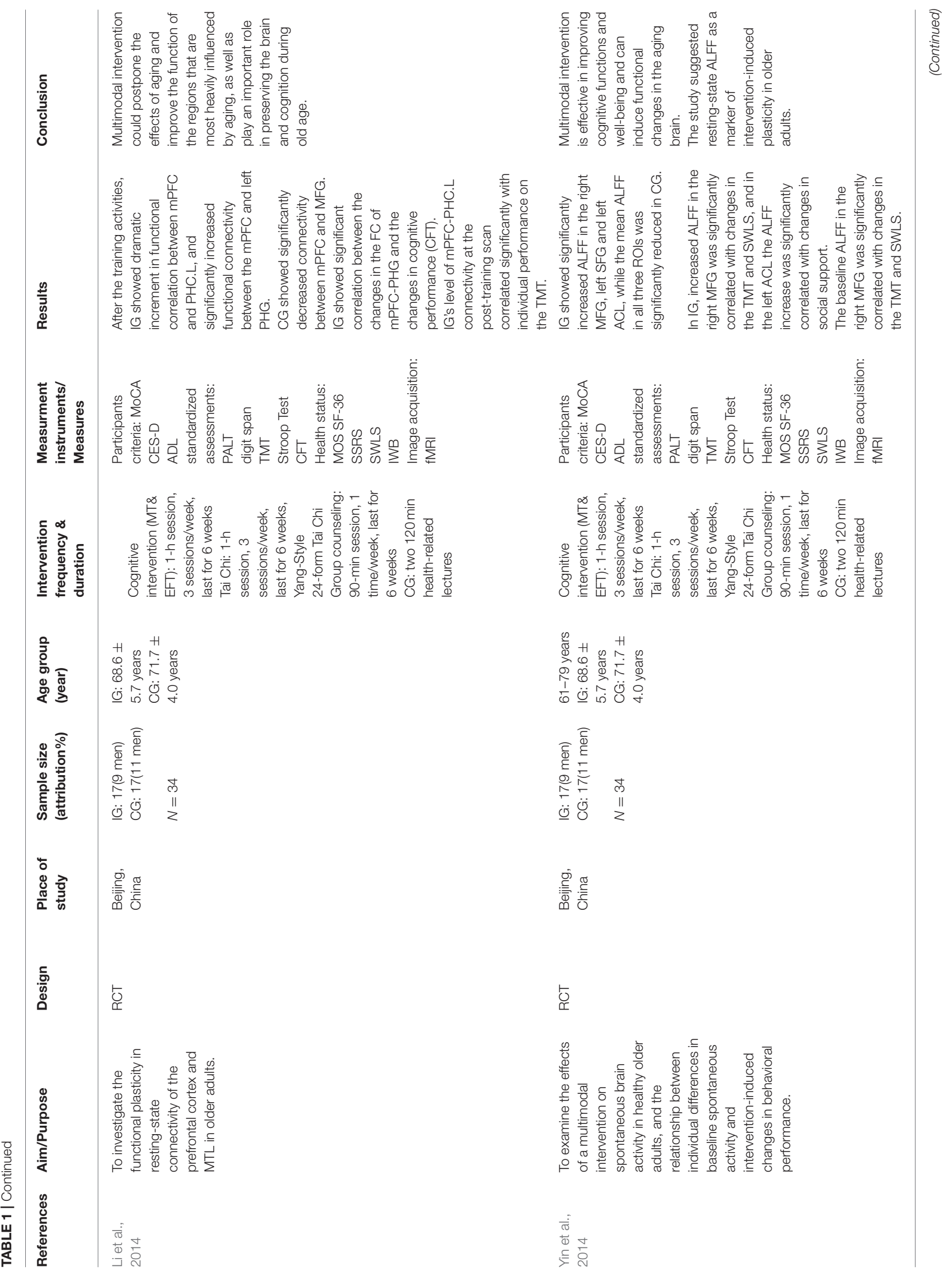




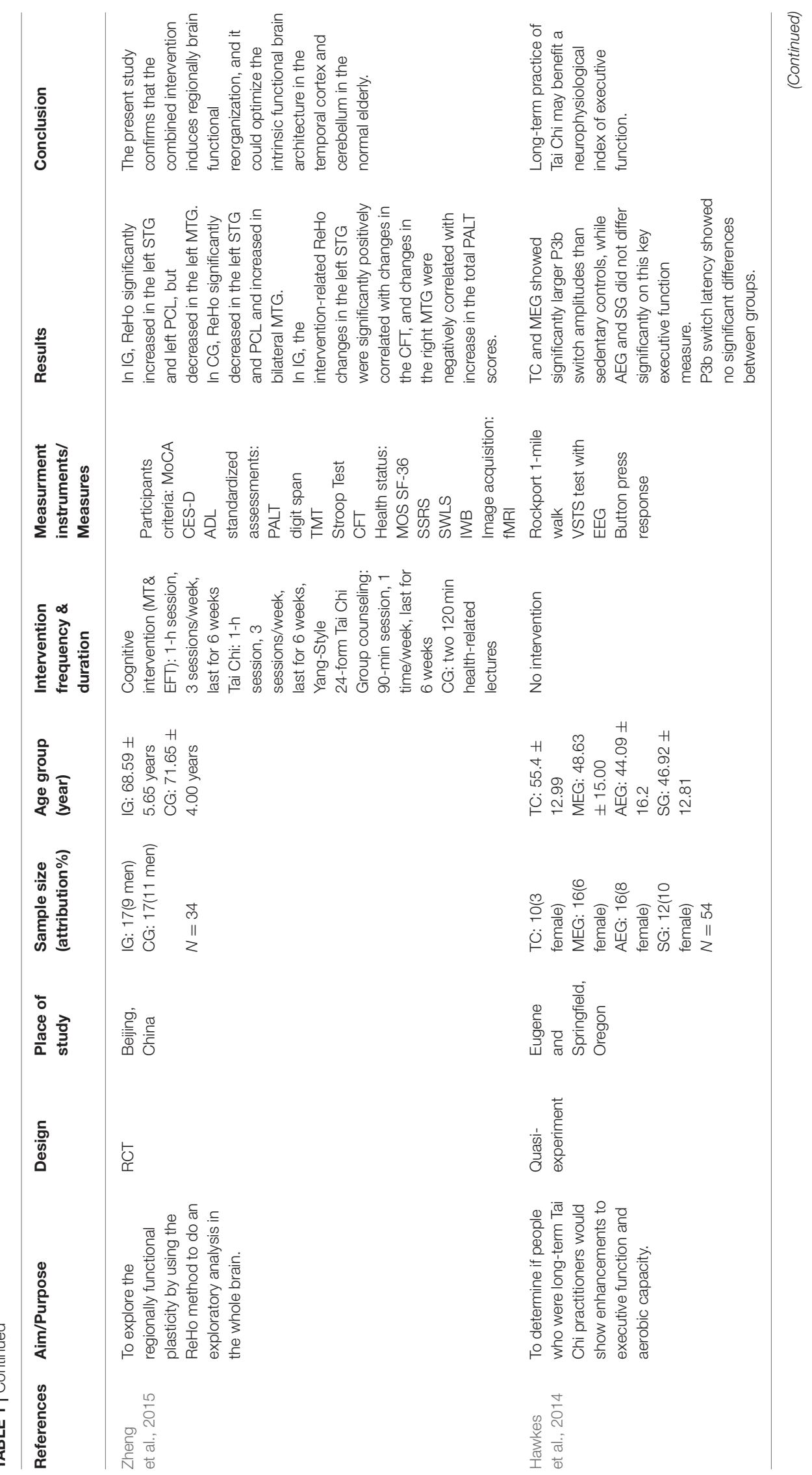




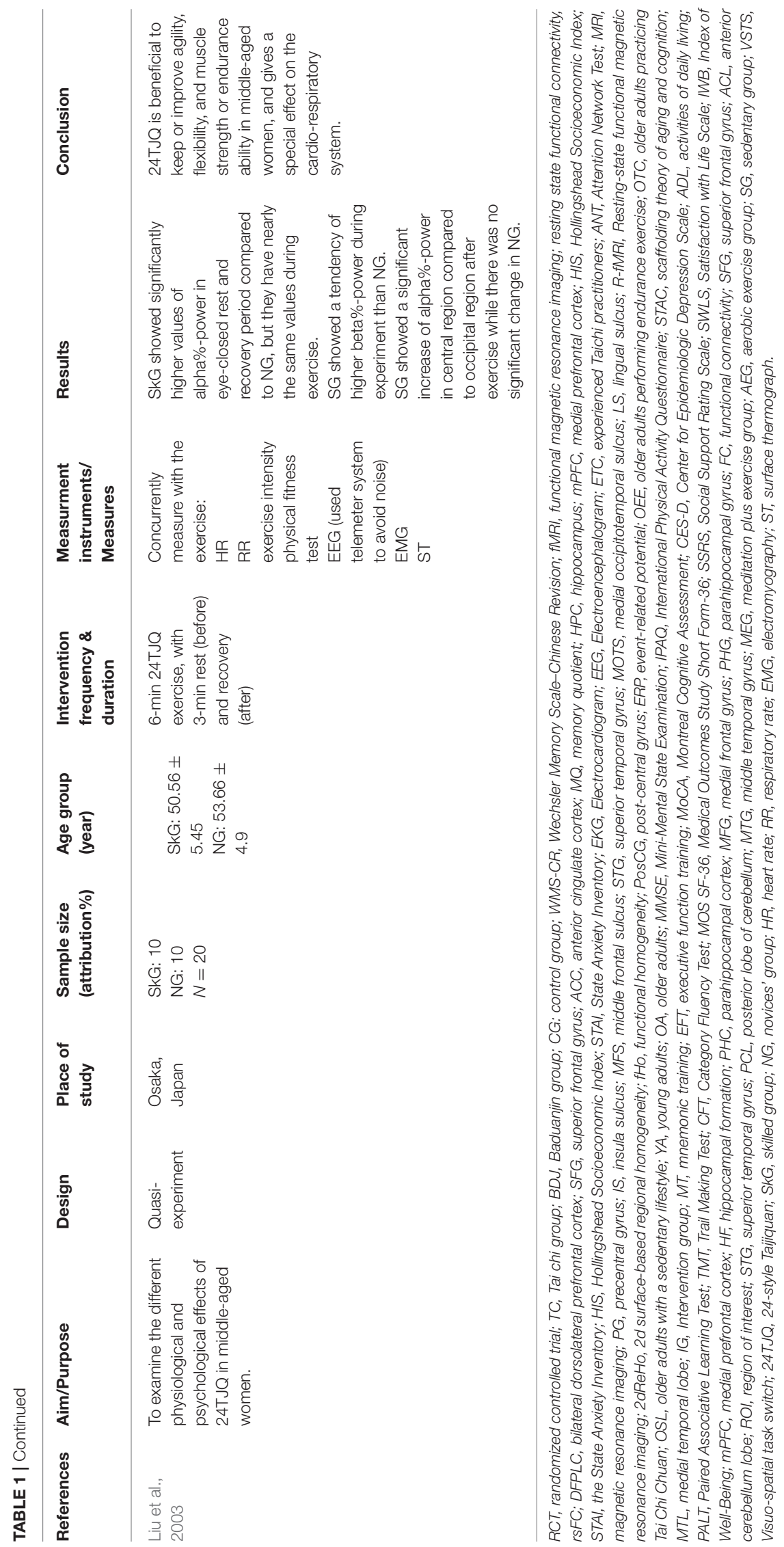


TABLE 2 | Quality assessment of reviewed studies.

\begin{tabular}{|c|c|c|c|c|c|c|c|c|c|c|c|c|}
\hline Study & EC & RA & CA & SAB & SB & TB & AB & DR & ITA & BC & PM & OSQ \\
\hline Tao et al., 2017 & Yes & Yes & Yes & Yes & Yes & Yes & Yes & No & Yes & Yes & Yes & High \\
\hline Tao et al., 2016 & Yes & Yes & Yes & Yes & Yes & Yes & Yes & No & Yes & Yes & Yes & High \\
\hline Field et al., 2010 & No & $C D$ & CD & $C D$ & No & Yes & $C D$ & Yes & Yes & NA & No & Low \\
\hline Wei et al., 2013 & Yes & No & No & NA & Yes & NA & Yes & Yes & NA & Yes & Yes & Moderate \\
\hline Wei et al., 2014 & Yes & No & No & NA & Yes & NA & Yes & Yes & NA & Yes & Yes & Moderate \\
\hline Fong et al., 2014 & Yes & No & No & NA & Yes & NA & Yes & Yes & NA & Yes & Yes & Moderate \\
\hline Li et al., 2014 & Yes & Yes & Yes & Yes & Yes & Yes & Yes & No & Yes & Yes & Yes & High \\
\hline Yin et al., 2014 & Yes & Yes & Yes & Yes & Yes & Yes & Yes & No & Yes & Yes & Yes & High \\
\hline Zheng et al., 2015 & Yes & Yes & Yes & Yes & Yes & Yes & Yes & No & Yes & Yes & Yes & High \\
\hline Hawkes et al., 2014 & Yes & No & No & NA & Yes & NA & Yes & Yes & NA & Yes & Yes & Moderate \\
\hline Liu et al., 2003 & Yes & No & No & NA & Yes & NA & Yes & Yes & NA & Yes & Yes & Moderate \\
\hline
\end{tabular}

EC, eligibility criteria; $R A$, random allocation; $C A$, concealed allocation; $S A B$, similar at baseline; SB, subject blinded; TB, therapist blinded; $A B$, assessor blinded; DR, drop-out rate; ITA, intention-to-treat analysis; BC, between-group comparison; PM, points measures; OSQ, overall study quality; CD, cannot determine; NA, not applicable.

connectivity included enhanced rsFC between the medial prefrontal cortex and the medial temporal lobe (Li et al., 2014). Given the fact that Tai Chi was the only form of physical activity in the intervention program, it is reasonable to assume that, to a certain extent, Tai Chi exercise contributed to the enhanced functional connectivity in correlation to improved cognitive performance.

Tao and colleagues examined correlates of mental control and functional connectivity (Tao et al., 2016, 2017). Participants who completed Tai Chi or a similar exercise (Baduanjin) over the 12-week intervention achieved a significant improvement in mental control and memory function. fMRI identified a significant decrease in the resting state functional connectivity (rsFC) between bilateral dorsolateral prefrontal cortex (DLPFC) and putamen, suggesting a negative relationship between mental control improvement and rsFC DLPFC-putamen connectivity (Tao et al., 2017). Superior memory function was found in alignment with increased rsFC between bilateral hippocampus and medial prefrontal cortex (Tao et al., 2016). Both studies substantiated the association between cognitive function and functional connectivity in prefrontal areas.

\section{Brain Neural Activity}

Regional homogeneity (ReHo) and amplitude of low-frequency fluctuations (ALFF) in BOLD signal of fMRI revealed spontaneous neuronal activity (Zang et al., 2004; Fox and Raichle, 2007). The previous study found that ALFF declines with aging (Zuo and Xing, 2014). A multimodal intervention including Tai Chi, cognitive training, and group counseling benefited the intervention group in which strengthened ALFF in the middle frontal gyrus, superior frontal gyrus, and anterior cerebellum lobe was observed (Yin et al., 2014). Another study following similar protocols identified reorganized ReHo in the superior and middle temporal gyri, and the posterior lobe of the cerebellum (Zheng et al., 2015). Enhanced intrinsic brain activity is the evidence of Tai Chi-induced benefits in promoting cognitive functions.

\section{Brain Electric Activity}

EEG detects brain electric activity, which is subject to physical activity. Participants showed better performance in math computation after Tai Chi and yoga practice (Field et al., 2010). Increased theta activity indicated immediate relaxation during exercise. The study suggested that Tai Chi and yoga exerted an immediate impact on brain activity. Brain plasticity was partially evident in that brain activity was adaptive to specific task.

Liu et al. (2003) investigated spontaneous brain activity of Tai Chi experts and novices during practice. Experts indicated a significantly higher alpha-wave amplitude than novices in eyeclose resting and recovery period, suggesting that the experts could quickly and effectively reach a psychological relaxation. Also, the experts exhibited a higher beta-wave amplitude than novices, implying that experts tend to be more physiologically excited than novices throughout the practice. Experts indicated well-developed mind concentration capacity, which was evident in the alpha shift tendency from occipital lobe to central or frontal regions.

Cognitive function was assessed by event-related potential (ERP) while subjects conducting a task-switch test under homogeneous and heterogeneous conditions (Fong et al., 2014). P3 amplitude exhibited no difference between young adults and older adults with either regular endurance training or Tai Chi exercise. However, all three groups indicated significantly larger P3 amplitude than that indicated in the group of sedentary older adults. Similar P3 patterns between young and older adults participating in long-term exercise provided evidence regarding the benefits of endurance training and Tai Chi exercise on cognitive function. Another study examining P3b amplitude of subjects conducting task-switch test confirmed the benefits of long-term Tai Chi practice in the neural substrates of executive function (Hawkes et al., 2014).

\section{DISCUSSION}

The included studies reported positive outcomes of Tai Chi practice in older adults. Specifically, Tai Chi-induced benefits 
involved with superior capacities in respect to mental control (Tao et al., 2017), memory (Tao et al., 2016), fitness (Liu et al., 2003; Wei et al., 2013), cognition (Fong et al., 2014; Li et al., 2014; Wei et al., 2014; Yin et al., 2014), and executive function (Field et al., 2010; Hawkes et al., 2014; Zheng et al., 2015). Findings as to physiological and psychological improvement substantiated the significant role of Tai Chi practice in counteracting age-related decline in motor and cognitive function. More importantly, neural imaging techniques applied to the included studies provided evidence on the connection between improved performance and changes in the neural system. Aging brain still retains some plasticity, which may contribute to delaying or reversing neurological deterioration in the aging process (Kramer et al., 2004; Gabbard, 2011). Wei et al. (2013) identified effects of Tai Chi intervention on reshaping brain structures. The finding is consistent with previous studies, which observed greater cortical thickness in older adults after memory training (Engvig et al., 2010), meditation practice (Lazar et al., 2005), and aerobic exercise (Colcombe et al., 2006). Functional change is associated with the development of new neurons and synapses in the brain (Honey et al., 2009; Cai et al., 2014). In alignment with other forms of exercise, Tai Chi exercise mitigates brain structural and functional deficits (Seidler et al., 2010). Older adults maintaining an active lifestyle by routinely practicing Tai Chi indicated enhanced neural plasticity (Liu et al., 2003; Field et al., 2010; Fong et al., 2014; Hawkes et al., 2014). The included studies provided evidence-based explanation on the neural mechanisms underlying the exerciseinduced improvement in motor and cognitive performance.

The reviewed studies only adopted tasks related to cognition, working memory, and executive function. Motor tasks, however, have yet been incorporated into EEG, fMRI, or MRI scan. In comparison to the EEG detection, which allows moderate physical activity, fMRI and MRI require subjects to maintain a resting state. Even small head motions may produce noise in brain scans (Power et al., 2012; Satterthwaite et al., 2012; Van Dijk et al., 2012), which proposed a challenge of integrate neuroimaging techniques into motor tasks. Researchers have designed a few tasks, which require a small range of motion such as finger tapping (Stoodley et al., 2012; Gardini et al., 2016), reaching and grasping (Culham et al., 2003), and lower limb joint motions (Kapreli et al., 2006). To expand knowledge on neural correlates of motor performance, feasible motor tasks should be developed to fit the setting of research employing the neuroimaging techniques.

Older adults experience reduced hemispheric asymmetry due to age-related deficits in neural connectivity (Cabeza, 2002). Evidence from fMRI scan indicated symmetric brain activations when older adults were performing cognitive tasks (Grady, 2000). A recent study involved with older adults also identified reduced asymmetry in movement patterns between dominant and non-dominant hand, suggesting a potential connection to the reduced hemispheric asymmetry
(Przybyla et al., 2011). However, the theory remains to be an assumption without direct evidence from a study, which applies fMRI to motor tasks. By investigating the change in motor performance, whether it is associated with age-related degeneration or Tai Chi-incurred improvement, researchers can better understand neural mechanisms underlying the aging process.

The lack of robust empirical research on Tai Chi-incurred changes for older adults is a limitation of the review. The inherent risk of bias in the study design, paired with the limited literature, suggests the necessity of an increasing number of RCTs in this field. Only two of the included studies reported effect size, which makes it difficult to compare the effectiveness between studies. Future research should report the effect size so that critical conclusion can be reached based on statistical evidence.

\section{CONCLUSION}

The literature review summarized 11 studies, which employed neuroimaging techniques and EEG to investigate effects of Tai Chi on hemispheric reorganization. The reviewed articles provide evidence that there may be cognitive improvement associated with modified brain activity, functional connectivity, and brain structures in older adults through Tai Chi exercise. Future studies should account for the potential connection between changed motor functions and corresponding neural mechanisms underlying the aging process. RCTs are needed to provide powerful evidence on the effect of Tai Chi intervention. In contrast to previous research focusing on performance, future studies should analyze the effects of intervention from the neurological standpoint. Applying neuroimaging techniques and EEG to Tai Chi intervention is worth investigating, which allows researchers to explore the neural mechanisms related to the effectiveness of Tai Chi exercise on counteracting the aging process.

\section{AUTHOR CONTRIBUTIONS}

$\mathrm{ZP}$ and $\mathrm{XS}$ contributed to the conception and design of the review. ZP, XS, and QF applied the search strategy. All authors applied the selection criteria. All authors completed assessment of risk of bias. All authors analyzed the data and interpreted data. $\mathrm{XS}, \mathrm{QF}$, and ZP wrote this manuscript. LH, YL, CC, JL, and M-LK critically edited the manuscript.

\section{FUNDING}

This research was supported by a combination of institutions as stated below: National Natural Science Foundation of China (NSFC31401018); The National Research Foundation of Korea Grant funded by the Korean Government (MOE) (NRF 2017S1A2A2039405); Mississippi State University College of Education Undergraduate Student Research Grant. 


\section{REFERENCES}

Adkins, D. L., Boychuk, J., Remple, M. S., and Kleim, J. A. (2006). Motor training induces experience-specific patterns of plasticity across motor cortex and spinal cord. J. Appl. Physiol. 101, 1776-1782. doi: 10.1152/japplphysiol.00515.2006

Cabeza, R. (2002). Hemispheric asymmetry reduction in older adults: the HAROLD model. Psychol. Aging 17, 85-100. doi: 10.1037/0882-7974.17.1.85

Cai, L., Chan, J. S., Yan, J. H., and Peng, K. (2014). Brain plasticity and motor practice in cognitive aging. Front. Aging Neurosci. 6:31. doi: 10.3389/fnagi.2014.00031

Colcombe, S. J., Erickson, K. I., Scalf, P. E., Kim, J. S., Prakash, R., McAuley, E., et al. (2006). Aerobic exercise training increases brain volume in aging humans. J. Gerontol. Ser. A Biol. Sci. Med. Sci. 61, 1166-1170. doi: 10.1093/gerona/61.11.1166

Colcombe, S. J., Kramer, A. F., Erickson, K. I., Scalf, P., McAuley, E., Cohen, N. J., et al. (2004). Cardiovascular fitness, cortical plasticity, and aging. Proc. Natl. Acad. Sci. U.S.A. 101, 3316-3321. doi: 10.1073/pnas. 0400266101

Culham, J. C., Danckert, S. L., De Souza, J. F., Gati, J. S., Menon, R. S., and Goodale, M. A. (2003). Visually guided grasping produces fMRI activation in dorsal but not ventral stream brain areas. Exp. Brain Res. 153, 180-189. doi: $10.1007 / \mathrm{s} 00221-003-1591-5$

Engvig, A., Fjell, A. M., Westlye, L. T., Moberget, T., Sundseth, Ø., Larsen, V. A., et al. (2010). Effects of memory training on cortical thickness in the elderly. Neuroimage 52, 1667-1676. doi: 10.1016/j.neuroimage.2010.05.041

Erickson, K. I., Colcombe, S. J., Wadhwa, R., Bherer, L., Peterson, M. S., Scalf, P. E., et al. (2007). Training-induced plasticity in older adults: effects of training on hemispheric asymmetry. Neurobiol. Aging 28, 272-283. doi: 10.1016/j.neurobiolaging.2005.12.012

Erickson, K. I., Gildengers, A. G., and Butters, M. A. (2013). Physical activity and brain plasticity in late adulthood. Dialogues Clin. Neurosci. 15, 99-108.

Field, T., Diego, M., and Hernandez-Reif, M. (2010). Tai chi/yoga effects on anxiety, heartrate, EEG and math computations. Complement. Ther. Clin. Pract. 16, 235-238. doi: 10.1016/j.ctcp.2010.05.014

Fong, D. Y., Chi, L. K., Li, F., and Chang, Y. K. (2014). The benefits of endurance exercise and Tai Chi Chuan for the task-switching aspect of executive function in older adults: an ERP study. Front. Aging Neurosci. 6:295. doi: 10.3389/fnagi.2014.00295

Fox, M. D., and Raichle, M. E. (2007). Spontaneous fluctuations in brain activity observed with functional magnetic resonance imaging. Nat. Rev. Neurosci. 8, 700-711. doi: 10.1038/nrn2201

Fox, M. D., Snyder, A. Z., Vincent, J. L., et al. (2007). Intrinsic fluctuations within cortical systems account for intertrial variability in human behavior. Neuron 56 , 171-184. doi: 10.1016/j.neuron.2007.08.023

Gabbard, C. P. (2011). Lifelong Motor Development. San Francisco, CA: Pearson Higher Ed.

Gardini, S., Venneri, A., McGeown, W. J., Toraci, C., Nocetti, L., Porro, C. A., et al. (2016). Brain activation patterns characterizing different phases of motor action: execution, choice and ideation. Brain Topogr. 29, 679-692. doi: 10.1007/s10548-016-0491-5

Giedd, J. N., Raznahan, A., Alexander-Bloch, A., Schmitt, E., Gogtay, N., and Rapoport, J. L. (2015). Child psychiatry branch of the National Institute of Mental Health longitudinal structural magnetic resonance imaging study of human brain development. Neuropsychopharmacology 40, 43-49. doi: $10.1038 /$ npp. 2014.236

Grady, C. L. (2000). Functional brain imaging and age-related changes in cognition. Biol. Psychol. 54, 259-281. doi: 10.1016/S0301-0511(00)00059-4

Hatta, A., Nishihira, Y., Kim, S. R., Kaneda, T., Kida, T., Kamijo, K., et al. (2005). Effects of habitual moderate exercise on response processing and cognitive processing in older adults. Jpn. J. Physiol. 55, 29-36. doi: 10.2170/jjphysiol.R2068

Hawkes, T. D., Manselle, W., and Woollacott, M. H. (2014). Tai Chi and meditation-plus-exercise benefit neural substrates of executive function: a cross-sectional, controlled study. J. Complement. Integr. Med. 11, 279-288. doi: 10.1515/jcim-2013-0031

Hoffstaedter, F., Grefkes, C., Roski, C., Caspers, S., Zilles, K., and Eickhoff, S. B. (2015). Age-related decrease of functional connectivity additional to gray matter atrophy in a network for movement initiation. Brain Struct. Funct. 220, 999-1012. doi: 10.1007/s00429-013-0696-2

Honey, C. J., Sporns, O., Cammoun, L., Gigandet, X., Thiran, J. P., Meuli, R., et al. (2009). Predicting human resting-state functional connectivity from structural connectivity. Proc. Natl. Acad. Sci. U.S.A. 106, 2035-2040. doi: 10.1073/pnas.0811168106

Jain, A., Taylor, J., Sanzo, P., and Zerpa, C. (2017). The effect of Tai Chi on functional lower extremity mobility and strength, ankle proprioception, and postural adaptation in older adults. American J. Med. Med. Sci. 7, 229-237. doi: 10.5923/j.ajmms.20170706.01

Kapreli, E., Athanasopoulos, S., Papathanasiou, M., Van Hecke, P., Strimpakos, N., Gouliamos, A., et al. (2006). Lateralization of brain activity during lower limb joints movement. An fMRI study. Neuroimage 32, 1709-1721. doi: 10.1016/j.neuroimage.2006.05.043

Kramer, A. F., Bherer, L., Colcombe, S. J., Dong, W., and Greenough, W. T. (2004). Environmental influences on cognitive and brain plasticity during aging. J. Gerontol. Ser. A Biol. Sci. Med. Sci. 59, M940-M957. doi: 10.1093/gerona/59.9.M940

Lam, L. C., Chau, R. C., Wong, B. M., Fung, A. W., Lui, V. W., Tam, C. C., et al. (2011). Interim follow-up of a randomized controlled trial comparing Chinese style mind body (Tai Chi) and stretching exercises on cognitive function in subjects at risk of progressive cognitive decline. Int. J. Geriatr. Psychiatry 26, 733-740. doi: 10.1002/gps.2602

Lazar, S. W., Kerr, C. E., Wasserman, R. H., et al. (2005). Meditation experience is associated with increased cortical thickness. Neuroreport 16:1893. doi: 10.1097/01.wnr.0000186598.66243.19

Li, R., Zhu, X., Yin, S., Niu, Y., Zheng, Z., Huang, X., et al. (2014). Multimodal intervention in older adults improves resting-state functional connectivity between the medial prefrontal cortex and medial temporal lobe. Front. Aging Neurosci. 6:39. doi: 10.3389/fnagi.2014.00039

Liu, Y., Mimura, K., Wang, L., and Ikuda, K. (2003). Physiological benefits of 24-style Taijiquan exercise in middle-aged women. J. Physiol. Anthropol. Appl. Human Sci. 22, 219-225. doi: 10.2114/jpa.22.219

Ni, M., Mooney, K., Richards, L., Balachandran, A., Sun, M., Harriell, K., et al. (2014). Comparative impacts of tai chi, balance training, and a speciallydesigned yoga program on balance in older fallers. Arch. Phys. Med. Rehabil. 95, 1620.e30-1628.e30. doi: 10.1016/j.apmr.2014.04.022

Paré, M., and Munoz, D. P. (2000). Immediate neural plasticity shapes motor performance. J. Neurosci. 20:RC52. doi: 10.1523/JNEUROSCI.20-01-j0005.2000

Petzinger, G. M., Fisher, B. E., Van Leeuwen, J. E., Vukovic, M., Akopian, G., Meshul, C. K., et al. (2010). Enhancing neuroplasticity in the basal ganglia: the role of exercise in Parkinson's disease. Mov. Disord. 25(Suppl. 1), S141-S145. doi: 10.1002/mds.22782

Power, J. D., Barnes, K. A., Snyder, A. Z., Schlaggar, B. L., and Petersen, S. E. (2012). Spurious but systematic correlations in functional connectivity MRI networks arise from subject motion. Neuroimage 59, 2142-2154. doi: 10.1016/j.neuroimage.2011.10.018

Przybyla, A., Haaland, K. Y., Bagesteiro, L. B., et al. (2011). Motor asymmetry reduction in older adults. Neurosci. Lett. 489, 99-104. doi: 10.1016/j.neulet.2010.11.074

Reid, K., Price, L., Harvey, W., et al. (2016). Changes in leg muscle strength and power after Tai Chi exercise in patients with symptomatic knee osteoarthritis. Osteoarthr. Cartil. 24:S430. doi: 10.1016/j.joca.2016.01.776

Satterthwaite, T. D., Wolf, D. H., Loughead, J., Ruparel, K., Elliott, M. A., Hakonarson, H., et al. (2012). Impact of in-scanner head motion on multiple measures of functional connectivity: relevance for studies of neurodevelopment in youth. Neuroimage 60, 623-632. doi: 10.1016/j.neuroimage.2011.12.063

Seidler, R. D., Bernard, J. A., Burutolu, T. B., Fling, B. W., Gordon, M. T., Gwin, J. T., et al. (2010). Motor control and aging: links to age-related brain structural, functional, and biochemical effects. Neurosci. Biobehav. Rev. 34, 721-733. doi: 10.1016/j.neubiorev.2009.10.005

Stoodley, C. J., Valera, E. M., and Schmahmann, J. D. (2012). Functional topography of the cerebellum for motor and cognitive tasks: an fMRI study. Neuroimage 59, 1560-1570. doi: 10.1016/j.neuroimage.2011.08.065

Tao, J., Chen, X., Egorova, N., Liu, J., Xue, X., Wang, Q., et al. (2017). Tai Chi Chuan and Baduanjin practice modulates functional connectivity of the cognitive control network in older adults. Sci. Rep. 7:41581. doi: 10.1038/srep41581 
Tao, J., Liu, J., Egorova, N., Chen, X., Sun, S., Xue, X., et al. (2016). Increased hippocampus-medial prefrontal cortex resting-state functional connectivity and memory function after tai chi chuan practice in elder adults. Front. Aging Neurosci. 8:25. doi: 10.3389/fnagi.2016.00025

Tomasi, D., and Volkow, N. D. (2012). Aging and functional brain networks. Mol. Psychiatry 17, 471, 549-558. doi: 10.1038/mp.2012.27

Tousignant, M., Corriveau, H., Roy, P. M., et al. (2013). Efficacy of supervised Tai Chi exercises versus conventional physical therapy exercises in fall prevention for frail older adults: a randomized controlled trial. Disabil. Rehabil. 35, 1429-1435. doi: 10.3109/09638288.2012.737084

Van Dijk, K. R., Sabuncu, M. R., and Buckner, R. L. (2012). The influence of head motion on intrinsic functional connectivity MRI. Neuroimage 59, 431-438. doi: 10.1016/j.neuroimage.2011.07.044

Verhagen, A. P., de Vet, H. C., de Bie, R. A., Kessels, A. G., Boers, M., Bouter, L. M., et al. (1998). The Delphi list: a criteria list for quality assessment of randomized clinical trials for conducting systematic reviews developed by Delphi consensus. J. Clin. Epidemiol. 51, 1235-1241. doi: 10.1016/S0895-4356(98)00131-0

Voss, M. W., Erickson, K. I., Prakash, R. S., Chaddock, L., Kim, J. S., Alves, H., et al. (2013). Neurobiological markers of exercise-related brain plasticity in older adults. Brain Behav. Immun. 28, 90-99. doi: 10.1016/j.bbi.2012.10.021

Wayne, P. M., Walsh, J. N., Taylor-Piliae, R. E., Wells, R. E., Papp, K. V., Donovan, N. J., et al. (2014). Effect of Tai Chi on cognitive performance in older adults: systematic review and meta-analysis. J. Am. Geriatr. Soc. 62, 25-39. doi: 10.1111 /jgs.12611

Wei, G. X., Dong, H. M., Yang, Z., Luo, J., and Zuo, X. N. (2014). Tai Chi Chuan optimizes the functional organization of the intrinsic human brain architecture in older adults. Front. Aging Neurosci. 6:74. doi: 10.3389/fnagi.2014.00074

Wei, G.-X., Xu, T., Fan, F.-M., et al. (2013). Can Taichi reshape the brain? A brain morphometry study. PLoS ONE 8:e61038. doi: 10.1371/journal.pone.0061038
Wong, A. M., Lin, Y. C., Chou, S. W., Tang, F. T., and Wong, P. Y. (2001). Coordination exercise and postural stability in elderly people: effect of Tai Chi Chuan. Arch. Phys. Med. Rehabil. 82, 608-612. doi: 10.1053/apmr.2001.22615

Yin, S., Zhu, X., Li, R., Niu, Y., Wang, B., Zheng, Z., et al. (2014). Interventioninduced enhancement in intrinsic brain activity in healthy older adults. Sci. Rep. 4:7309. doi: 10.1038/srep07309

Zang, Y., Jiang, T., Lu, Y., He, Y., and Tian, L. (2004). Regional homogeneity approach to fMRI data analysis. Neuroimage 22, 394-400. doi: 10.1016/j.neuroimage.2003.12.030

Zheng, Z., Zhu, X., Yin, S., Wang, B., Niu, Y., Huang, X., et al. (2015). Combined cognitive-psychological-physical intervention induces reorganization of intrinsic functional brain architecture in older adults. Neural Plast. 2015:713104. doi: 10.1155/2015/713104

Zuo, X. N., and Xing, X. X. (2014). Test-retest reliabilities of restingstate FMRI measurements in human brain functional connectomics: a systems neuroscience perspective. Neurosci. Biobehav. Rev. 45, 100-118. doi: 10.1016/j.neubiorev.2014.05.009

Conflict of Interest Statement: The authors declare that the research was conducted in the absence of any commercial or financial relationships that could be construed as a potential conflict of interest.

Copyright (C) 2018 Pan, Su, Fang, Hou, Lee, Chen, Lamberth and Kim. This is an open-access article distributed under the terms of the Creative Commons Attribution License (CC BY). The use, distribution or reproduction in other forums is permitted, provided the original author(s) and the copyright owner are credited and that the original publication in this journal is cited, in accordance with accepted academic practice. No use, distribution or reproduction is permitted which does not comply with these terms. 